7th International Workshop on Astronomy and

Relativistic Astrophysics (IWARA 2016)

International Journal of Modern Physics: Conference Series

Vol. 45 (2017) 1760015 (4 pages)

(C) The Author(s)

DOI: $10.1142 / \mathrm{S} 2010194517600151$

\title{
Jefimenko's Gravito-Electromagnetic Equations as an Implication of the Helmholtz Theorem, Adapted to Time-Varying Vector Fields
}

\author{
Fabrício T. Dalmolin, César H. Lenzi \\ Departamento de Física \\ Universidade Tecnológica Federal do Paraná (UTFPR) \\ Av. Brasil, 4232, P. O. Box 271, 85884-000 Medianeira, PR, Brazil \\ ftdalmolin@gmail.com, chlenzi1980@gmail.com \\ Orimar A. Battistel \\ Departamento de Física \\ Universidade Tecnológica Federal do Paraná (UTFPR) \\ Av. Brasil, 4232, P. O. Box 271, 85884-000 Medianeira, PR, Brazil \\ Universidade Federal de Santa Maria (UFSM) \\ 97105-900 Santa Maria, RS, Brazil \\ orimar.battistel@gmail.com
}

Published 15 August 2017

\begin{abstract}
Using the Helmholtz theorem (modified to time-varying vector fields) the Jefimenko's gravito-electromagnetic equations are deduced. Newton's gravitational field emerges as a particular case when an action at a distance theory is considered.
\end{abstract}

Keywords: Gravito-Electromagnetic Equations.

PACS numbers: 03.50.-z, 03.65.Ca, 04.20.Cv, 04.25.Nx

\section{Helmholtz Theorem in the Lorenz Gauge}

One of the most important theorems in classical field theories is the Helmholtz theorem for vector fields, ${ }^{1}$ which states that any sufficiently smooth, rapidly decaying vector field in three dimensions can be written as the sum of two parts: an irrotational vector field represented by the gradient of a scalar potential $\nabla \phi$ and another one, the solenoidal part, represented by a curl of a vector potential $\boldsymbol{\nabla} \times \boldsymbol{A}$.

The Helmholtz theorem is usually applied to write and solve the field equations in electrostatics and magnetostatics. However, in order to write the field equations in

This is an Open Access article published by World Scientific Publishing Company. It is distributed under the terms of the Creative Commons Attribution 4.0 (CC-BY) License. Further distribution of this work is permitted, provided the original work is properly cited. 
the presence of a time-dependent vector field is necessary to consider two auxiliary functions as

$$
\phi(\boldsymbol{r}, t)=\int \frac{f\left(\boldsymbol{r}^{\prime}, t-\frac{\left|\boldsymbol{r}-\boldsymbol{r}^{\prime}\right|}{c_{g}}\right)}{4 \pi\left|\boldsymbol{r}-\boldsymbol{r}^{\prime}\right|} d^{3} V^{\prime}
$$

and

$$
\boldsymbol{A}(\boldsymbol{r}, t)=\int \frac{\boldsymbol{\Gamma}\left(\boldsymbol{r}^{\prime}, t-\frac{\left|\boldsymbol{r}-\boldsymbol{r}^{\prime}\right|}{c_{g}}\right)}{4 \pi\left|\boldsymbol{r}-\boldsymbol{r}^{\prime}\right|} d^{3} V^{\prime},
$$

that represents the retarded potentials to the sources $f(\boldsymbol{r}, t)$ (scalar source) and $\boldsymbol{\Gamma}(\boldsymbol{r}, t)$ (vector source) in close analogy with the electromagnetism. Here, $c_{g}$ represents the field propagation speed in free space.

From the functions represented in Eqs.(1) and (2) is possible to show that:

$$
\left(\nabla^{2}-\frac{1}{c_{g}^{2}} \frac{\partial^{2}}{\partial t^{2}}\right) \phi(\boldsymbol{r}, t)=-f(\boldsymbol{r}, t)
$$

and

$$
\left(\nabla^{2}-\frac{1}{c_{g}^{2}} \frac{\partial^{2}}{\partial t^{2}}\right) \boldsymbol{A}(\boldsymbol{r}, t)=-\boldsymbol{\Gamma}(\boldsymbol{r}, t),
$$

which is of course the wavelike solutions to the sources. The associated sources are defined as

$$
f(\boldsymbol{r}, t)=-4 \pi G \rho
$$

and

$$
\boldsymbol{\Gamma}(\boldsymbol{r}, t)=-4 \pi G \boldsymbol{J} / c_{g} .
$$

Considering the double curl vector identity for the vector potential as follows

$$
\boldsymbol{\nabla} \times \boldsymbol{\nabla} \times \boldsymbol{A}=\boldsymbol{\nabla}(\boldsymbol{\nabla} \cdot \boldsymbol{A})-\nabla^{2} \boldsymbol{A},
$$

and applying some modifications

$$
\boldsymbol{\nabla} \times \boldsymbol{\nabla} \times \boldsymbol{A}=\boldsymbol{\nabla}\left(-\frac{1}{c_{g}} \frac{\partial \phi}{\partial t}\right)-\left(\frac{4 \pi G}{c_{g}} \boldsymbol{J}+\frac{1}{c_{g}^{2}} \frac{\partial^{2} \boldsymbol{A}}{\partial t^{2}}\right),
$$

then Eq.(6) can be conveniently rewrite as

$$
-\frac{4 \pi G}{c_{g}} \boldsymbol{J}=\frac{1}{c_{g}} \frac{\partial}{\partial t}\left(\boldsymbol{\nabla} \phi+\frac{1}{c_{g}} \frac{\partial \boldsymbol{A}}{\partial t}\right)+\nabla \times \nabla \times \boldsymbol{A},
$$

which is nothing more than of the Helmholtz theorem, modified for time-varying vector fields. ${ }^{3}$ Eq. (7) is obtained using the Lorenz gauge represent by

$$
\frac{1}{c_{g}} \frac{\partial \phi(\boldsymbol{r}, t)}{\partial t}=-\boldsymbol{\nabla} \cdot \boldsymbol{A}(\boldsymbol{r}, t) \text {. }
$$

This step ensures the Lorenz gauge condition within the Helmholtz theorem ${ }^{\mathrm{a}}$.

${ }^{a}$ For a detailed deduction of the gravito-electromagnetic equations see Gravito-electromagnetic equations from the Helmholtz theorem, adapted to time-varying vector fields. ${ }^{2}$ 
From Eqs. (3) and (4) and its sources in the retarded time is possible to write the scalar potential and the vector potential respectively as

$$
\phi(\boldsymbol{r}, t)=-G \int \frac{\rho\left(\boldsymbol{r}^{\prime}, t-\frac{\left|\boldsymbol{r}-\boldsymbol{r}^{\prime}\right|}{c_{g}}\right)}{\left|\boldsymbol{r}-\boldsymbol{r}^{\prime}\right|} d V^{\prime},
$$

and

$$
\boldsymbol{A}(\boldsymbol{r}, t)=-\frac{G}{c_{g}} \int \frac{\boldsymbol{J}\left(\boldsymbol{r}^{\prime}, t-\frac{\left|\boldsymbol{r}-\boldsymbol{r}^{\prime}\right|}{c_{g}}\right)}{\left|\boldsymbol{r}-\boldsymbol{r}^{\prime}\right|} d V^{\prime} .
$$

Note that the scalar source $(f(\boldsymbol{r}, t))$ is the only one experimental input. All the others equations are consequence of the approach.

\section{Jefimenko's Gravito-Electromagnetic Equations}

From Eq.(7), the time-dependent gravitational field $\boldsymbol{g}(\boldsymbol{r}, t)$ and the time-dependent co-gravitational field $\boldsymbol{K}(\boldsymbol{r}, t)$, as named by Jefimenko, ${ }^{4}$ can be defined in analogy with the electromagnetism as follows:

$$
\begin{gathered}
-\nabla \phi-\frac{1}{c_{g}} \frac{\partial \boldsymbol{A}}{\partial t}=\boldsymbol{g}(\boldsymbol{r}, t), \\
\boldsymbol{\nabla} \times \boldsymbol{A}=\boldsymbol{K}(\boldsymbol{r}, t) .
\end{gathered}
$$

The Eqs.(11) and (12) for the potentials yields the Jefimenko's equations for fields $\boldsymbol{g}(\boldsymbol{r}, t)$ and $\boldsymbol{K}(\boldsymbol{r}, t)$. In order to obtain the first one in terms of their sources and its time variations, the retarded scalar potential Eq.(9) can be rewrite as

$$
\phi(\boldsymbol{r}, t)=-G \int \frac{\rho\left(\boldsymbol{r}^{\prime}, \tau\right)}{R} d V^{\prime},
$$

where $\left|\boldsymbol{r}-\boldsymbol{r}^{\prime}\right| \equiv R$ and $t-R / c_{g} \equiv \tau$ are conveniently defined as done for the electromagnetic case in the reference. ${ }^{3}$

Using the definition given by Eq.(11) the field equation becomes

$$
\boldsymbol{g}(\boldsymbol{r}, t)+\frac{1}{c_{g}} \frac{\partial \boldsymbol{A}(\boldsymbol{r}, t)}{\partial t}=G \int \boldsymbol{\nabla}\left(\frac{\rho\left(\boldsymbol{r}^{\prime}, \tau\right)}{R}\right) d V^{\prime} .
$$

It is easy to show that

$$
\nabla \rho=-\frac{1}{c_{g}} \frac{\partial \rho}{\partial \tau} \hat{e}_{R}
$$

and

$$
\nabla\left(\frac{1}{R}\right)=-\frac{1}{R^{2}} \hat{e}_{R}
$$


Finally the Jefimenko's equation to the time-dependent gravitational field is

$$
\boldsymbol{g}(\boldsymbol{r}, t)=-G \int\left(\frac{\rho}{R^{2}}+\frac{1}{c_{g} R} \frac{\partial \rho}{\partial \tau}\right) \hat{e}_{R} d V^{\prime}+\frac{G}{c_{g}^{2}} \int \frac{1}{R} \frac{\partial \boldsymbol{J}}{\partial \tau} d V^{\prime} .
$$

Similarly to performed for the $\boldsymbol{g}(\boldsymbol{r}, t)$ field and knowing that the $\boldsymbol{K}(\boldsymbol{r}, t)$ field is solenoidal is possible to write from the vector potential Eq.10

$$
\boldsymbol{K}(\boldsymbol{r}, t)=-\frac{G}{c_{g}} \int\left(\frac{\boldsymbol{J}}{R^{2}}+\frac{1}{R c_{g}} \frac{\partial \boldsymbol{J}}{\partial \tau}\right) \times \hat{e}_{R} d V^{\prime} .
$$

In your book Gravitation and Co-gravitation, ${ }^{4}$ Jefimenko starts his generalization of Newton's theory from the Eqs.(17) and (18). Newton's Gravitational field can be obtained as a particular case when the Eqs.(17) and (18) are placed in the scenario of an action at a distance theory $\left(c_{g} \rightarrow \infty\right)$,

$$
\boldsymbol{g}(\boldsymbol{r})=-G \int \frac{\rho}{R^{2}} \hat{e}_{R} d V^{\prime}
$$

\section{Acknowledgments}

F. T. D. acknowledges support from Universidade Tecnológica Federal do Paraná (UTFPR).

\section{References}

1. G. B. Arfken, Mathematical Methods for Physicists (Academic Press, Washington, 1970).

2. F. T. Dalmolin, C. H. Lenzi, and O. A. Battistel Submitted, October (2016).

3. M. Davis Am. J. Phys., vol. 74, 72 (2006).

4. O. D. Jefimenko, Gravitation and Co-gravitation. (Electret Scientific Company, Star City, 2006). 